\title{
The Research of Risk Transport Chain of Power Industry Carbon Emissions Trading Costs
}

\author{
Yu Xiaobao ${ }^{1, a^{*}}$, Tan Zhongfü, b \\ ${ }^{1}$ North China Electric Power University, Beijing 102206, China \\ aemail:yuxiaobao1222@163.com, \\ bemail:tanzhongfubeijing@126.com
}

Keywords: Carbon emissions trading, History predicts, Transport chain, Optimization.

Abstract. A great many uncertain factors affect the cost of carbon emissions trading, and they interact to form a complex system. To improve the accuracy of prediction, it is necessary to deepen the study of these influencing factors. Through investigation this paper identified 21 main factors affecting the cost of carbon emissions trading. By processing and interpretation of the structural model analysis, this paper constructed of carbon emissions trading costs and the impact of class structure factors transport chain. Take into account all the factors affecting the cost of carbon emissions trading, to improve the accuracy of prediction of improving related infrastructure. Take some areas for example, the paper compared error rate of prediction between the historical forecasting method and delivery chain method, the results indicate that the latter error rate lower, the average degree of optimization can be achieved $24.35 \%{ }^{1}$

\section{Introduction}

As a large carbon emissions industry, power industry is inevitable to consider the problem of carbon emissions trading cost in the process of forming trading market. The forecast of carbon emissions trading cost is the basis to control the trading costs reasonably, which directly relate to the economic development of the power industry and environment impacts. Domestic carbon emission trading market is still in its infancy, the current method for predicting the cost of carbon emissions trading is basically blank, just to fit the available estimates based on historical values, which lead to a large prediction deviation. And it may affect the economic development of power industry. Due to the mutual influence of factors that affect the cost of carbon emissions trading, there is a complex influence system. This paper studied on the relationship between these factors, and figured out the impact of these factors on the cost of carbon emissions trading, which have a great contribution to economic development of power industry.

\section{Analysis model of impact factors of carbon emissions trading cost}

Time series prediction method is an extension of the historical data to predict, also known as an extension of the historical prediction. In this method, a time series could reflect the socio-economic development process and the regularity of the phenomenon were an extension of extrapolation methods to predict trends. Due to less research of carbon emissions trading costs, the change in carbon emissions trading costs cannot be determined, therefore we use linear trend model, to predict future values based on the principle of least square method.

We can get the influencing factors hierarchical structure diagram and transport chain of power industry carbon emissions trading cost through ISM model, and establish the trading cost predicting correction model of power industry carbon emissions based on the model:

$$
C=C_{0}+\Delta C=C_{0}+\sum_{j=1}^{m} f_{j}\left(\Delta x_{j}\right)
$$

\footnotetext{
${ }^{1}$ This paper is funded by Project of Beijing Graduates Cultivation of Combination of Production, Teaching and Research.
} 
In the formula, $C$ is the forecasting value of power industry carbon emissions trading cost based on influence transport chain. $C_{0}$ is the historical forecasting value of carbon emissions trading cost without the effect of influence transport chain. $\Delta C$ is the value of influence transport chain affect the carbon emissions cost $j=1,2, \ldots, m$ is for the influence transport chains which affect the carbon emissions trading cost. $\Delta x_{j}$ is the changing value of different influence source index. $f_{j}\left(\Delta x_{j}\right)$ is the carbon emissions cost influence function of different influence sources. Influence function can be decided by the different influence transport chain which effects the carbon emissions trading cost and the experience of experts.

\section{The establishment of the ISM model of carbon emissions trading cost}

There are many factors affecting the carbon emissions trading cost. In recent years, economy scale, energy intensity, energy structure and industrial structure have the highest frequency of occurrence as the carbon emission influence factors when studying the relevant changing rules. And the technology and efficiency are also seen as the focus which reducing the carbon emission by many scholars. This article determines the factors that influence the carbon emissions trading cost based on the Delphi method, and ultimately get 21 factors impact on the carbon emissions trading costs (S17): carbon emissions (S1), population (S2), GDP (S3), power consumption (S4), electricity consumption growth (S5), electricity demand elasticity (S6), carbon intensity (S7), the proportion of thermal power (S8), thermal power generation (S9), coal consumption amount (S10), oil consumption (S11) gas consumption (S12), the power industry carbon emissions (S13), carbon emissions trading price (S14), carbon emissions trading credits (S15) emissions initial quota (S16), technical progress (S18), vegetation uptake (S19), the vegetation coverage area (S20), vegetation destruction rate (S21), the per capita demand for wood (S22).

Here article reference literature Analysis of Carbon Emissions Trading Costs Influencing Factors in Power Industry. From the case, the factor with a strong driving force is the key factor. Therefore, we can order the factors in reachable matrix $\mathrm{R}$ by the factors driving force of each rows from small to large, from top to bottom, then order the column factors in sequence with row factors to get the reordering matrix. According to the reordering reachable matrix, the factors with same driving force can be considered as the same hierarchical structure factor; then, use arrow to connect the related factors box. We can get the Interpretative Structure Model.

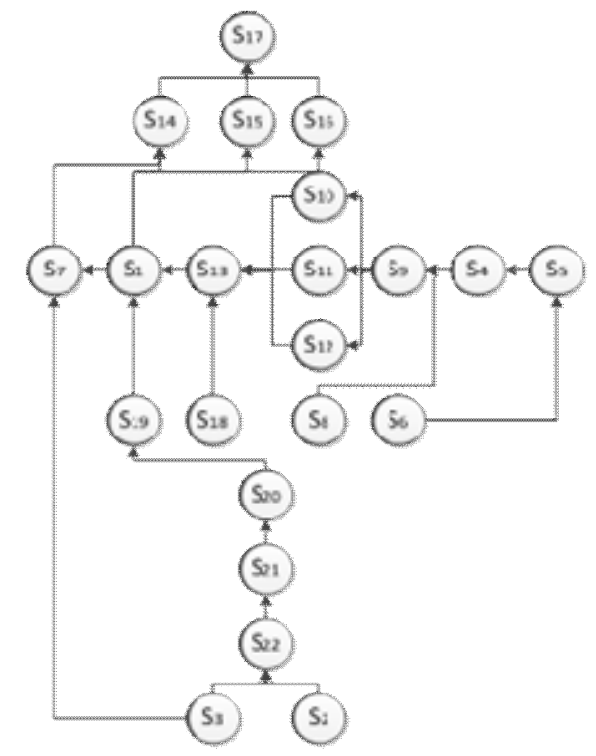

Fig. 1 The hierarchical structure of influence factors of carbon emissions trading cost

Thus, according to the analysis of the influence factors of ISM in power industry, we can divide above 22 influencing factors into eight levels. These 22 risk factors of eight levels make up the different influence transport chain from top to bottom. According to the different location in the influence 
transport chain, we can ultimately divided the 22 influence factors into the end effect (17), the source effects $(2,3,6,8,18)$ and the process effects (except the end effect and source effects). The end effect can be only one, namely carbon emission trading cost in power industry; the process effects are the intermediate link from the source to end which can not only affect the end effects through the upper level indirectly, but also affect the end effects directly. Source effects are the root of causing the end effects. The population (2), GDP (3) in the 8th level, the electricity demand elasticity (6), thermal power ratio (8), and technical progress (18) in the fourth level are all source effects from different impact of carbon emissions trading costs in power industry.

Seeing from the influence factors hierarchy of carbon emission trading cost, the influence factors which affect carbon emission trading cost in power industry make up the different influence transport chain. The factors will eventually have an impact on carbon emissions trading cost in power industry. The influencing transport chains are as Figure 3 shown. This paper divided the transport chain into five based on different effect sources including: economic conditions influence transport chain, GDP per capita influence transport chain, electricity demand elasticity influence transport chain, power structure influence transport chain and technical progress influence transport chain.

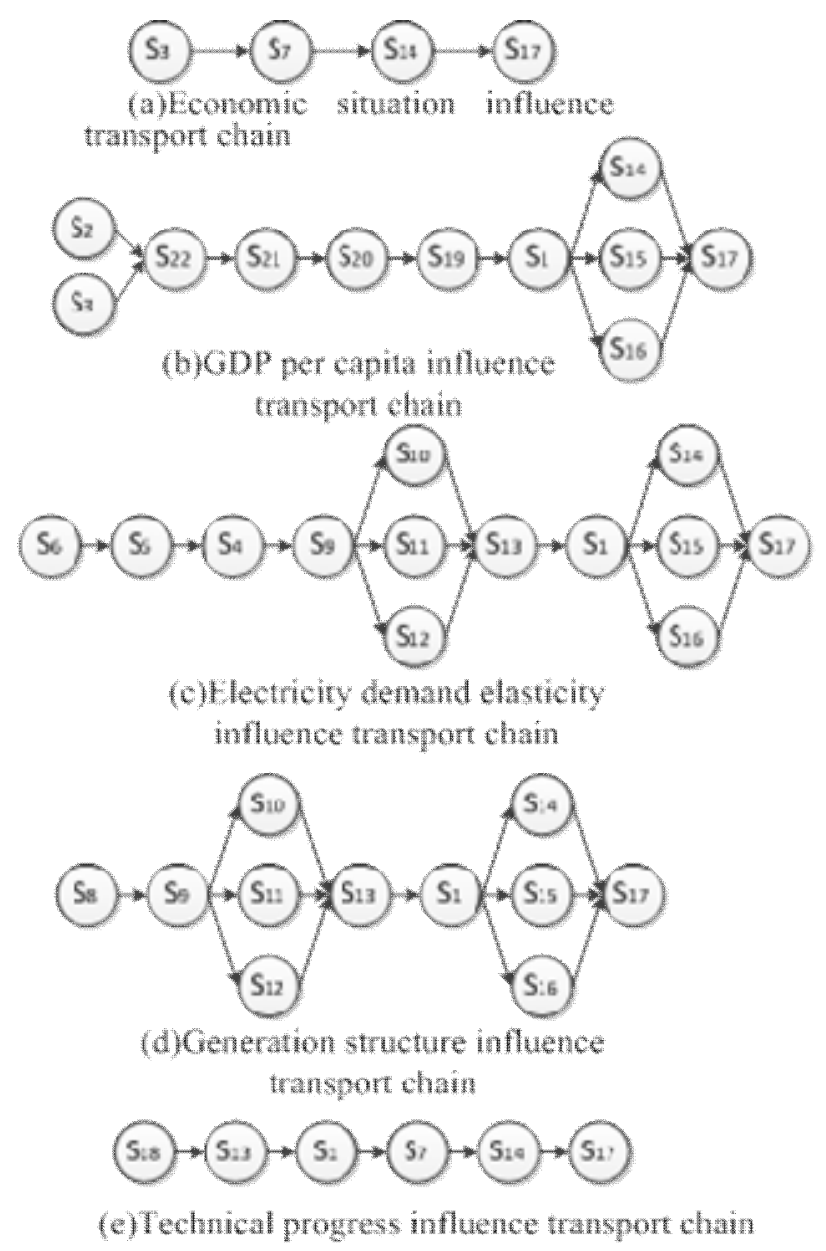

Fig. 2 Influence transport chain of carbon emissions trading cost in power industry

As economic situation influence transport chain for example, this paper try to determine the influence function that each transport chains impact on the electricity consumption. The impact of economic situation influence transport chain on the carbon emissions trading cost has a faster economic development. And the greater the demand for electricity is, the more carbon emissions is, the greater the carbon emissions trading cost is. This article uses the following formula to represent the impact of economic situation influence transport chain on the carbon emissions trading cost.

$$
f_{1}(\Delta G)=\lambda_{g} \frac{\Delta G}{G_{0}} C_{0}
$$


In the formula, $f_{1}(\Delta G)$ is an influence function that $\Delta G$ affecting the electricity industries carbon emissions trading cost. $\Delta G$ is the changes value of GDP growth. $G_{0}$ is the GDP in the base year without considering $\Delta G . \lambda_{g}$ is the correlation coefficient between carbon emission trading cost and GDP growth. $C_{0}$ is the carbon emissions trading cost without considering the impact of transport chain.

In this article, it should be stressed that the influence functions of each transport chain are not unique, but rather on the basis of the objective laws for various types risk and the feasibility principle of quantitative calculation. These functions are mostly based on the expert opinions and the author's cognitive experience. Now the carbon emissions trading market in China has built up initially, there are few data about carbon emissions trading cost.

\section{Analysis of examples}

Take a region for example to verify the feasibility of the transport chain forecasting method. Statistic data in the area over the years, including: carbon emissions trading costs, GDP, demand for wood, electricity consumption elasticity coefficient, the proportion of thermal power and carbon intensity.

First, according to statistics of the calendar year of carbon emissions trading cost data, using historical forecasting method to predict, been predicted curve shown in Figure 3, fitting curve 1.

Secondly, according to the transport chain forecasting method to predict, been predicted curve, shown in Figure 3, fitting curve 2.

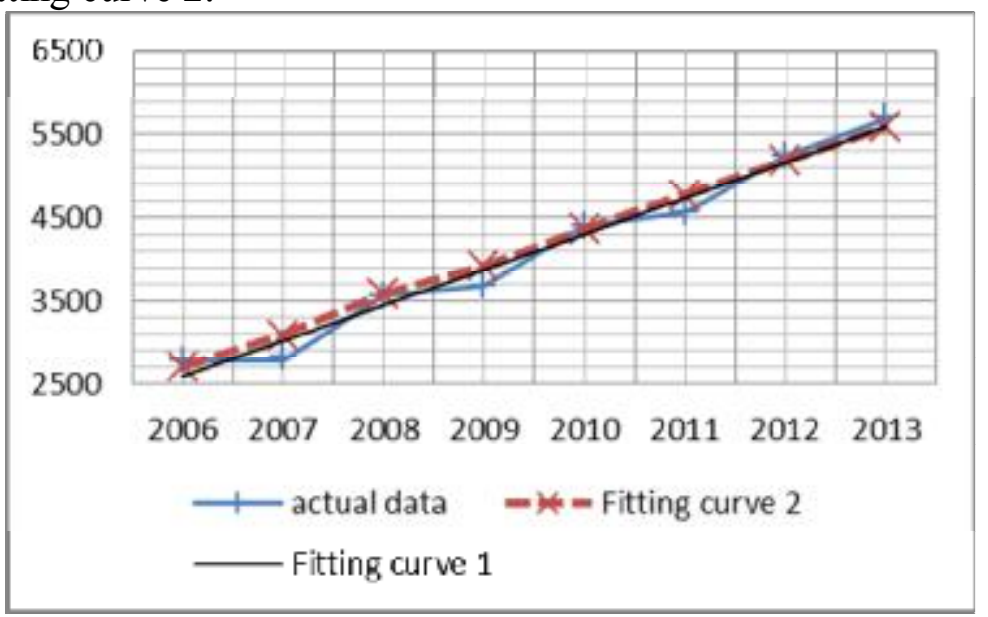

Fig.3 Fitting curve of history of carbon emissions trading cost

Statistics the error rate of two forecasting methods based on calculations, as shown in Table 1.

Table 1 Error rate statistics of different methods

\begin{tabular}{cccc}
\hline error rate & historical forecasting method & transfer chain forecasting method & degree of optimization \\
\hline 2006 & -0.03909 & -0.02555 & 0.34636 \\
2007 & 0.097244 & 0.099265 & -0.02078 \\
2008 & -0.01945 & 0.00216 & 0.888949 \\
2009 & 0.060957 & 0.065082 & -0.06766 \\
2010 & -0.01907 & -0.011 & 0.423148 \\
2011 & 0.038591 & 0.046836 & -0.21366 \\
2012 & -0.01788 & -0.01094 & 0.388516 \\
2013 & -0.02431 & -0.01936 & 0.203689
\end{tabular}

As can be seen from Table 1, the transfer chain forecasting method has obvious advantages compared to historical prediction method, the average degree of optimization has reached 24.35 percent, to optimize the degree of predictive value in 2008 even reached $89 \%$. Thus, the proposed transfer chain forecasting method is scientific and rational, and higher than the accuracy of the historical prediction method. 


\section{Conclusion}

With the launch of carbon trading market, it is more and more important to study the carbon emissions trading cost in the future, and the uncertainty influence factors of carbon emissions trading costs are increasing. In order to improve the accuracy of the forecasts, the influence factors should have further research. This article confirms 21 main influencing factors through investigating. Based on the Interpretative Structure Model, we constructed the hierarchical structure of influence factors of carbon emissions trading cost and the influence transport chains, and built up the prediction model of carbon emissions trading cost. This article makes an innovation from the view of analysis, and takes into account all factors that affect the carbon emissions trading cost to improve the accuracy of predictions.

\section{References}

[1] Zhang, Yue-Jun; Wang, Ao-Dong; Da, Ya-Bin Regional allocation of carbon emission quotas in China: Evidence from the Shapley value method. ENERGY POLICY. 2014,74:454-464

[2] Yang Guorui. A Study on the Fluctuation of Carbon Emission in China's Economic Development and the Path of Reducing Carbon Emissions [D]. A Dissertation Submitted in Partial Fulfillment of the Requirements for the Degree of Philosophy in Economics. 2010.05

[3] He Yongxiu, Tao Weijun, Yang Weihong, Dai Aiying, CAI Qi, Furong LI. Urban Power Load Forecasting Based on an Interpretative Structural Model [J]. Automation of Electric Power Systems. 2009 (33): 37-42

[4] Wu Yunna, Bian Qing. Analysis of Affecting Factors of Cost Control of Wind Power Construction Project Based on ISM[J]. Journal of Wuhan University of Technology. 2012 (2): 257-261

[5] He Hong Bing. Factors Analysis of Influencing China's Carbon Emissions[D]. A Dissertation Submitted in Partial Fulfillment of the Requirements for the Degree of Philosophy in Economics. 2012.05

[6] Zhang Youguo. Economic Development Pattern Change Impact on China's Carbon Intensity [J]. Economic Research Journal. 2010 (04): 120-133

[7] Zhu Qin, Peng Xizhe, Lu Zhiming, Wu Kaiya. Factors Decomposition and Empirical Analysis of Variations in Energy Carbon Emission in China [J]. Resource Sciences. 2009 (12): 2072-2079

[8] Zhu Qin, Peng Xizhe, Lu Zhiming, Juan Yu. Analysis Model and Empirical Study of Impacts from Population and Consumption on Carbon Emissions [J]. China Population Resources and Environment,. 2012 (02): 98-102

[9] Li Jian, Zhou Hui. Correlation Analysis of Carbon Emission Intensity and Industrial Strccture in China [J]. China Population Resources and Environment. 2012 (01): 7-14 